East African Medical Journal Vol. 81 No. 4 April 2004

MYCOBACTERIAL ADENITIS: ROLE OF MYCOBACTERIUM BOVIS, NON-TUBERCULOUS MYCOBACTERIA, HIV INFECTION, AND RISK FACTORS IN ARUSHA, TANZANIA

S.G.M. Mfinanga, MD., Mphil, Centre for International Health, University of Bergen, Norway N-5021 Bergen Norway, National Institute for Medical Research (NIMR), Muhimbili Station, O. Morkve, MD, PhD, Centre for International Health,University of Bergen, Norway N-5021 Bergen Norway, R. R. Kazwala, BVsc, MVM, PhD, Sokoine University of Agriculture (SUA), Box 3021 Morogoro, Tanzania, S. Cleaveland, Bsc, MA, Vet MB, PhD., Centre for Tropical Veterinary Medicine, University of Edinburgh, Easter Bush, Roslin, Midlothian EH25 9RG UK., J. Kunda MD., Moredun Research Institute, 408 Gilmerton Road, Edinburgh EHI 7 7JH, UK., M. J. Sharp, BVMS, PhD, Moredun REsearch Institute, 408 Gilmerton Road Edinburgh EH17 7 JH, United Kingdom and R. Nilsen, DMD, PhD., Centre for International Health (CIH), University of Bergen, Norway N-5021 Bergen Norway, National Institute for Medical Research (NIMR), Muhimbili Station, Box 3436 Dar-es-Salaam, Tanzania

Request for reprints to: Dr. S.G.M. Mfinanga, Centre for International Health, University of Bergen, Norway N-5021 Bergen Norway and National Institute for Medical Research (NIMR), Muhimbili Station, P.O Box 3436, Dar-es-Salaam, Tanzania

\title{
MYCOBACTERIAL ADENITIS: ROLE OF MYCOBACTERIUM BOVIS, NON-TUBERCULOUS MYCOBACTERIA, HIV INFECTION, AND RISK FACTORS IN ARUSHA, TANZANIA
}

\author{
S.G.M. MFINANGA, O. MORKVE, R. R. KAZWALA, S. CLEAVELAND, \\ M. J. SHARP, J. KUNDA and R. NILSEN
}

\begin{abstract}
Objective: To assess risk factors and mycobacterial agents in mycobacterial adenitis. Design: Cross sectional involving comparison analysis of high-risk groups. Setting: Seven hospitals in rural and semi-rural districts of Arusha. Subjects: The study comprised of 457 patients of clinically diagnosed mycobacterial adenitis. Interventions: Biopsy materials were cultured and identification of mycobacterial isolates, and HIV infection testing were performed using standard methods. A questionnaire was used to establish information for assessing risk factors.

Main outcome measures: Proportions of mycobacterial isolates, risk factors and odds ratios.

Results: Of the 457 specimens, $65(14.2 \%)$ were culture positive. Isolates identified were M. bovis, 7(10.8\%) M. tuberculosis, $27(41.5 \%)$ and non-tuberculous mycobacteria 31(47.7\%). HIV infection and ingestion of raw milk were linked with increased risk of $M$. bovis infection by OR of $13.6(95 \% \mathrm{CI}, 1.7$ - 109.9) and 15.28 (3.26 - 71.7), respectively. On multivariate analysis, an OR of $16.2(1.3$ - 201.3) for having $M$. bovis adenitis was linked to HIV infection, raw milk and houses with poor ventilation. An OR of 5.2 (1.2 - 20.6) for non-tuberculous mycobacterial adenitis was linked to history of TB in the family, HIV infection, raw milk, raw animal products and poor knowledge on transmission of tuberculosis.

Conclusions: $M$. bovis caused one out of ten cases of culture positive mycobacterial adenitis. Non-tuberculous mycobacteria were more common than $M$. tuberculosis (50\% and $40 \%$ of the cases, respectively). HIV infection and raw animal products are among the risk factors identified for $M$. bovis and non-tuberculous mycobacterial adenitis.
\end{abstract}

\section{INTRODUCTION}

Tuberculosis (TB) is the leading cause of death due to a single infectious agent among adults in the world(1). Whereas the progress in global TB control caused by $M$. tuberculosis has been increasing (WHO report, $\mathrm{WHO} / \mathrm{CDS} / \mathrm{TB} / 2001.287)$, there is little information on the disease caused by $M$. bovis in developing countries. Risk factors in both animals and humans are present in tropical areas(1). HIV infection may perpetuate the $M$. bovis cycle of transmission of animal to human, human to human and human to animal(2). Consequently, the prevalence of human tuberculosis due to $M$. bovis infection has the potential of increasing(3). Of all nations in Africa, only seven apply disease control measures in animals as part of test and slaughter policy. This policy requires veterinary public personnel to carry out tuberculin testing on cattle, and to slaughter infected animals. The remaining 48 countries control the disease inadequately or not at all, and 33 have reported $M$. bovis in animals(1). $M$. bovis infection in man usually results in extrapulmonary tuberculosis, in particular cervical lymphadenitis(4). In countries where tuberculosis in cattle is common, about $10 \%$ of human tuberculosis cases are assumed to be due to M. bovis(3). However, in most cases, mycobacterial adenitis is caused by $M$. tuberculosis(4).

Non-tuberculous mycobacteria infection has been linked with cervicofacial manifestations in childhood(5). In recent years, environmental mycobacteria (EM) disease has become particularly relevant to human health due to the high prevalence of EM in pulmonary disease affecting patients with AIDS. In addition, the 
EM infection affects the immune responses to BCG vaccination(6). The EM are opportunistic causes of disease in humans and animals(6). Unlike tuberculosis, infections with non-tuberculous mycobacteria are acquired from the environment, where they are widely distributed in soil, water and food(7).

Human tuberculosis is a main cause of human morbidity and mortality in Tanzania (Ministry of Health $(\mathrm{MoH})$, healthy statistics abstract, 1999: 7(1); National Tuberculosis, and Leprosy Control Program \{NTLP annual reports, (1984 -2000). The number of cases increased from 11,753 in 1983 to 54,513 in 2000, with about $15-20 \%$ of all TB cases reported as extrapulmonary tuberculosis. In Tanzania, disproportionately high numbers of mycobacterial adenitis cases are reported in Arusha and Mbeya regions, where most of the populations are subsistence farmers and livestock keepers (NTLP annual reports 1984 -2000). This suggests that zoonotic transmission of tuberculosis may have a role in human extra-pulmonary TB in Tanzania.

The HIV epidemic has had a strong influence on the tuberculosis epidemic(8). The effect of HIV infection to M. bovis disease in the country is not known. The NTLP depends on examination of sputum specimens for AFB microscopy and some for culture to identify M. tuberculosis. However, lymph node specimens are not collected on routine bases, and the processing of sputum specimens does not include tests which are essential for isolation of M. bovis. Contrary to pulmonary tuberculosis, there have been limited studies on the role of mycobacteria adenitis and epidemiology of $M$. bovis on tuberculosis morbidity. M. bovis, however, has been isolated in cases of cervical adenitis(9). More studies are essential to provide more information on its contribution to the morbidity of tuberculosis. This article describes the role of $M$. bovis and nontuberculous mycobacteria as causes of mycobacterial adenitis, and related risk factors.

\section{MATERIALS AND METHODS}

Study areas and personnel training: The study was carried out in Mbulu, Babati and Hanang districts in Arusha region. The enrollment of patients was done from 1999 through 2001, in seven hospitals of the four districts. The hospitals were Karatu, Oldian, Haydom, Mbulu, Kateshi, Dareda, and Babati. Research personnel were Districts Tuberculosis and Leprosy Co-ordinators (DTLCs) or clinical officers who are responsible for tuberculosis in the seven hospitals. The DTLCs and the officers participated in a three day seminar to standardise study procedures. During the seminar, the clinical questionnaire for the study was reviewed to make sure that the DTLCs and clinical officers understood how to fill the questionnaire. One day was used for training on biopsy procedures and storage of specimens. Electric and kerosene deep freezers were supplied for storage of specimens. When necessary, the training was continued during supervision visits.

Patients enrollment and study procedures: Patients who enrolled in the study were those diagnosed clinically with mycobacterial adenitis according to national guidelines (Manual of the NTLP 1995). The guidelines are also recommended by the WHO (WHO report, WHO/CDS/TB/2001.287). The DTLCs and clinical officers interviewed the patients and filled in the study questionnaires.

Collection of biopsy and serum specimens: The biopsy specimens were taken from all patients fulfilling the diagnostic guidelines before starting anti-TB chemotherapy. The specimens were placed in universal containers and stored in the supplied deep-freezers. The DTLCs and clinical officers conducted pre-testing counselling for HIV testing and obtained verbal consent before drawing blood for HIV testing. Patients were assured that the results would be handled confidently and when they were reluctant, the specimens were not collected. The sera were separated from blood, and labelled NUNC tubes (NUNC, Intermed, Denmark) were used for storage in deep-freezers. The NUNC tubes were labelled with identification numbers to ensure the confidentiality of the results.

Culture, identification, and HIV test procedures: The frozen biopsy specimens were processed for culture and identification tests at the Central Tuberculosis Reference Laboratory. All processing of samples were carried out using aseptic techniques in a safety cabinet. The processing involved cutting the samples into tiny pieces and grinding them thoroughly by using pestle and mortar. The tissue suspensions were transferred into sterile universal containers. For decontamination process, $3 \%$ oxalic acid was added to the suspensions and left for 30 minutes while being shaken intermittently. The homogenates made were then centrifuged at $3000 \mathrm{rpm}$ for 15 minutes and supernatants were decanted into a $5 \%$ phenol disinfectant. To neutralise the sediments, $2 \%$ sodium hydroxide solution, which contained 3-4 drops of $0.1 \%$ phenol red indicator was added. The neutralisation was achieved when suspensions colour changed from yellow to orange-red. The suspensions were then centrifuged and the sediments inoculated on blood agar and on two types of Lowenstein Jensen media. Two loopfuls of the sediments were inoculated on each of the two Lowenstein Jensen media, one media contained glycerin and another pyruvate. The blood agar was incubated at $37^{\circ} \mathrm{C}$ overnight. If contaminant grew, the decontamination procedure was repeated and processing time increased to one hour. The Lowenstein Jensen media were incubated at $37^{\circ} \mathrm{C}$ for 6 to 12 weeks while observing for signs of growth on weekly bases.

Established protocols $(10,11)$ were used to identify mycobacteria species. The observation of isolates with eugenic growth on both Lowenstein Jensen media were tentatively identified as $M$. tuberculosis while those with eugenic growth in the pyruvate containing media were regarded as suggestive of $M$. bovis. The chemical identification tests according to Grange and Collins(10,11) were further carried out for identification of mycobacteria species. Growth on Lowenstein media containing PNB $(500 \mu \mathrm{g} / \mathrm{ml} 4$ paranitrobenzoic acid) were considered to be non-tuberculous mycobacteria. Other biochemical tests according to Grange and Collins $(10,11)$ included niacin test, nitrate reduction test, oxygen preference test, and twin 80 test.

Sera were tested for HIV infection using single Behring ELISA tests (Dade Behring Marburg GmbH, Emil-vonBehring Marburg/Germany) for negative and repeated test for positive results using Wellcozyme HIV Recombinant (Murex Biotech/UK). The tests were conducted according to manufacture's protocol. 
Data management and statistical analysis: Data management and analysis were carried out using Epi-Info Version 6.4 and SPSS 9.0 for Windows software(12). Recoding procedure was done to allow computer entries of responses for open questions and combinations of multiple responses to respective questions in the clinical forms. The variables were categorised to form groups of interest for comparison analysis.

The knowledge on spread of pulmonary tuberculosis was categorised as "good" if the patients knew that TB could be spread by air droplets from infected persons and "poor" if did not. The knowledge of bovine TB spread was categorised as "good" if the patients were aware that TB could be spread from infected animals to man and "poor" if not. The status of milk was categorised as "boiled" if both fresh and soured milk were boiled, and "not boiled" if one or both types of milk were not boiled. The housing ventilation was categorised as "good" if the houses had at least one window and "poor" if they had a hole or nothing for ventilation.

Stratification and logistic regression analysis were carried out to assess and adjust for interactions and potential cofounders of risk factors for mycobacterium isolates. The adjusted variables are shown in footnotes of respective Tables. Pearson Chi-squares and Wald statistics were used to compare group differences of categorical variables. Culture negatives adenitis cases were used as a comparison group for culture positivity, M. bovis, M. tuberculosis, and nontuberculous mycobacteria. Differences were considered statistically significant if $\mathrm{p} \leq 0.05$.

Multivariate logistic regression modelling was used to assess risk factors for the mycobacterium isolates. After screening with stratification analysis, nine variables were considered in multivariate analysis. The procedure used was backward stepwise selection that based on the probability of the likelihood-ratio statistic. During the modelling, a significance level of 0.1 was used as cut off value to eliminate a variable from the model. The variables in the models were tested and adjusted for interactions. The models were selected basing on goodness of fit statistics, model Chi-squares and significance of $95 \%$ confidence intervals for the adjusted odds ratios. In addition, the selection considered the clinical and epidemiological importance of the risk factors in the models.

Ethical issues: The project proposal was reviewed and approved by the Medical Research Co-ordinating Committee (MRCC) in Tanzania. Verbal consent was obtained from each patient enrolled in the study. The patients were explained the study purpose and counselled on the laboratory tests for specimens collection. Only those who accepted on their own will were included in the study. All patients tested for HIV infection were pre and post-test counselled. For confidentiality, laboratory forms for HIV testing were labelled with identification numbers only, and one investigator handled the results. All laboratory results were communicated to the responsible medical personnel for management of the patients.

Conflict of interest statement: There is no conflict of interest related to this article. The funding sources, namely Norwegian Research Council, Norway and Department for International Development, UK, had not influenced judgments of authors.

\section{RESULTS}

Baseline characteristics of culture results: Table 1 shows the baseline characteristics of culture results for the study patients. Of 457 analysed specimens, $14.2 \%(65 / 457)$ were culture positive. Hanang had a statistically higher proportion of culture positives patients $(23.1 \%, 15 / 65$, Wald statistic $=6.84, \mathrm{p}=0.009)$ than the other districts. Patients from the Barabaig tribe had a statistically higher proportion of culture positive specimens than other tribes $(11.7 \%, 7 / 60$, Wald statistic $=6.84, \mathrm{p}=0.009$ ).

Risk factors for culture positive mycobacterial adenitis: Table 2 shows risk factors for adenitis patients that were culture positive for tuberculosis. Only patients from the Barabaig tribe (5/6) that drank raw milk showed significant risk for culture positive mycobacterial adenitis (OR 4.55, 95\%CI, 1.41-14.66). Patients with the habit of eating raw animals' products (meat and offal) from Mbulu $(26.7 \%, 4 / 15)$ and from Iraqw tribe $(33.3 \%, 12 / 36)$ had higher risk for culture positive tuberculosis (OR 4.3, 95\%CI 1.8-10.5 and OR 2.86, 95\% CI 1.6 - 5.3, respectively).

Mycobacterium isolates and associated risk factors: Table 3 shows mycobacterium isolates and the associated risk factors. Of 65 culture positive specimens, the isolates were seven $(10.8 \%)$ M. bovis, $27(41.5 \%) \mathrm{M}$. tuberculosis, and 31(47.7\%) non-tuberculous mycobacteria. A. bovis adenitis cases were associated with HIV infection and drinking raw milk. HIV positive persons had an increased risk (OR 13.6, 95\% CI 1.7109.9) of being infected with A. bovis. Ingesting raw milk was also linked with increased risk of $M$. bovis infection (OR 15.3, 95\% CI, 3.3-71.7). The cases of adenitis caused by non-tuberculous mycobacteria (35.7\%, $10 / 28$ ) were associated with eating raw animal products like blood, meat, and offal (OR 3.5, 95\% CI, 1.5 - 7.9).

Multiple risk factors for culture positives and Mycobacterium isolates: Table 4 gives findings on the modelled assessments of multiple risk factors for mycobacterium isolates. The assessments showed sets of risk factors, which were associated with the isolates. The Chi-squares for Goodness-of-fit tests (HosmerLemeshow) for all the models were not statistically significant, and therefore, the models described the data reasonably well. Poor knowledge on transmission of TB from animals to humans in a patient with history of TB in the family showed a significant risk for culture positive adenitis. The OR for the two risk factors was 3.5(95\% CI, 1.4-9.0). A patient with HIV infection and drinking raw milk had an OR of 23.7 (95\% CI, 1.9 -299.3) for adenitis due to M. bovis. Poor knowledge on transmission of TB from animals to humans in a 
patient with history of TB in the family were related to non-tuberculous mycobacteria cases (OR 4.5, 95\% CI, 1.3 - 15.4). A set of five risk factors had an Odds Ratio of 4.2 (95\% CI, 1.3-13.8) for culture positive TB patients. The risk factors were, drinking raw milk, eating raw animal products (blood, meat, and offal),
HIV infection and, poor knowledge on transmission of TB from animals to humans in a patient with history of $\mathrm{TB}$ in the family. A significant risk for A. bovis adenitis was linked to drinking raw milk and living in houses with poor ventilation in a patient with HIV infection.

Table 1

The baseline characteristics of culture results from mycobacteria adenitis patients

\begin{tabular}{|c|c|c|c|}
\hline \multirow[t]{2}{*}{ Characteristic } & Culture Positive & \multicolumn{2}{|c|}{ Culture negative } \\
\hline & $(\%)$ & No. & $(\%)$ \\
\hline Culture results $(n=457)$ & (65/457) 14.2 & $(392 / 457)$ & 85.8 \\
\hline \multicolumn{4}{|l|}{ Gender $(n=444)$} \\
\hline Male & $(32 / 65) \quad 49.2$ & $(184 / 379)$ & 48.5 \\
\hline Female & $(33 / 65) \quad 50.8$ & $(195 / 374)$ & 51.5 \\
\hline \multicolumn{4}{|l|}{$\operatorname{Age}^{a} \quad(n=444)$} \\
\hline $1-19$ & $(22 / 65) \quad 33.8$ & $(165 / 379)$ & 43.5 \\
\hline $20-39$ & $(32 / 65) \quad 49.3$ & $(150 / 379)$ & 39.6 \\
\hline$>39$ & $\begin{array}{ll}(11 / 65) & 16.9\end{array}$ & $(64 / 379)$ & 16.9 \\
\hline \multicolumn{4}{|l|}{ Districts $(n=451)$} \\
\hline Babati & $(32 / 65) \quad 49.2$ & $(169 / 386)$ & 43.8 \\
\hline Mbulu & $(15 / 65) \quad 23.1$ & $(97 / 386)$ & 25.1 \\
\hline Hanang & $(15 / 65)^{\mathrm{b}} 23.1$ & $(80 / 386)$ & 20.7 \\
\hline Karatu & $(3 / 65) \quad 4.6$ & $(40 / 386)$ & 10.4 \\
\hline \multicolumn{4}{|l|}{ Diagnostic Units $(n=453)$} \\
\hline Babati & $(21 / 65) \quad 32.3$ & $(84 / 388)$ & 21.6 \\
\hline Dareda & $\begin{array}{ll}(11 / 65) & 16.9\end{array}$ & $(105 / 388)$ & 27.1 \\
\hline Hanang & $\begin{array}{ll}(7 / 65) & 10.8\end{array}$ & $(31 / 388)$ & 8.0 \\
\hline Mbulu & $(3 / 65)$ & $(11 / 388)$ & 2.8 \\
\hline Haydom & $\begin{array}{ll}(22 / 65) & 33.8\end{array}$ & $(125 / 388)$ & 32.2 \\
\hline Karatu & $(0)$ & $(9 / 388)$ & 2.4 \\
\hline Oldian & $(1 / 65)$ & $(231388)$ & 5.9 \\
\hline \multicolumn{4}{|l|}{ Occupation $(\mathrm{n}=298)$} \\
\hline Livestock only & $(7 / 44)$ & $(62 / 254)$ & 24.4 \\
\hline Traditional Farmer only & $(25 / 44) \quad 56.8$ & $(104 / 254)$ & 41.0 \\
\hline Livestock and farming & (12/44) 27.3 & $(77 / 254)$ & 30.3 \\
\hline Others $^{c}$ & (0) & $(11 / 254)$ & 4.3 \\
\hline \multicolumn{4}{|l|}{ Tribes $(n=415)$} \\
\hline Iraqw & $(38 / 60) \quad 63.3$ & $(249 / 355)$ & 70.1 \\
\hline Barabaig & $(7 / 60)^{b} \quad 11.7$ & $(21 / 355)$ & 5.9 \\
\hline Nyaturu & $(3 / 60) \quad 5.0$ & $(6 / 355)$ & 1.7 \\
\hline Datoga & $(3 / 60)$ & $(31 / 355)$ & 8.7 \\
\hline Rangi & $(1 / 60)$ & $(11 / 355)$ & 3.2 \\
\hline Others ${ }^{\mathrm{d}}$ & $(8 / 60)$ & $(37 / 355)$ & 10.4 \\
\hline
\end{tabular}

* Does not add up to 468 because, 10 specimens were contaminated, and one not done because quantity was not sufficient. aAdjusted by districts. ${ }^{b}$ District adjusted by tribe and vice versa, Wald statistic $=6.84, \mathrm{df}=1, \mathrm{p}=0.009, \mathrm{OR}=5.18(95 \% \mathrm{CI}$, 1.5-17.78). ${ }^{\mathrm{C}}$ Teachers, medical personnel, veterinary personnel, hunters. ${ }^{\mathrm{d}}$ Other tribes were from regions, which were not neighbouring to Arusha. 
Table 2

Risk factors for culture positive mycobacteria adenitis

\begin{tabular}{|c|c|c|c|c|c|c|}
\hline \multirow[t]{2}{*}{ Risk factor* } & \multicolumn{2}{|c|}{ Culture positive } & \multicolumn{2}{|c|}{ Culture negative } & \multicolumn{2}{|c|}{ OR $(95 \%$ C.I $)$} \\
\hline & No. & $(\%)$ & No. & $(\%)$ & & \\
\hline HIV Positive & $(5 / 29)$ & 17.2 & $(13 / 124)$ & 10.5 & $(0.68-3.57)$ & 1.56 \\
\hline History of $\mathrm{TB}$ in family & $(31 / 62)$ & 50.0 & $(168 / 360)$ & 46.7 & $(0.71-1.77)$ & 1.12 \\
\hline Poor ventilation & $(14 / 62)$ & 22.6 & $(115 / 362)$ & 31.8 & $(0.87-1.02)$ & 0.94 \\
\hline Livestock in the same house & $(13 / 41)$ & 31.7 & $(120 / 275)$ & 46.4 & $(0.34-1.19)$ & 0.64 \\
\hline Drinking unboiled milk & $(19 / 57)$ & 33.3 & $(89 / 350)$ & 25.4 & $(0.81-2.67)$ & 1.46 \\
\hline Barabaig & $(5 / 6)$ & 83.3 & $(8 / 18)$ & 44.4 & $(1.41-14.66)^{+}$ & 4.55 \\
\hline Uncooked animals' products** & $(17 / 60)$ & 28.3 & $(49 / 356)$ & 13.8 & $(1.27-3.44)^{+}$ & 2.06 \\
\hline Mbulu & $(4 / 15)$ & 26.7 & $(4 / 88)$ & 4.5 & $(1.77-10.50)^{+}$ & 4.32 \\
\hline Iraqw & $(12 / 36)$ & 33.3 & $(30 / 246)$ & 12.2 & $(1.55-5.26)^{+}$ & 2.86 \\
\hline \multicolumn{7}{|l|}{ Adenitis sites } \\
\hline Cervical & $(52 / 62)$ & 83.9 & $(306 / 359)$ & 89.1 & $(0.37-1.26)$ & 0.69 \\
\hline Poor knowledge & $(17 / 62)$ & 27.4 & $(103 / 353)$ & 29.2 & $(0.55-1.56)$ & 0.93 \\
\hline TB spread by air drops & $(37 / 60)$ & 61.7 & $(240 / 339)$ & 70.8 & $(0.44-1.14)$ & 0.71 \\
\hline
\end{tabular}

${ }^{*}$ The risk factors stratified by district and tribes. ${ }^{+}$Statistically significant results, Only statistically significant results of stratification analysis are reported. Odd Ratio of HIV infection was adjusted for age and gender. ${ }^{* *}$ Blood, meat and offal

Table 3

Mycobacterium isolates from adenitis patients and their risk factors

\begin{tabular}{|c|c|c|c|c|c|c|c|c|}
\hline \multirow{2}{*}{$\begin{array}{l}\text { Risk factor* /isolate } \\
\text { distribution }\end{array}$} & \multicolumn{2}{|c|}{ M. bovis } & \multicolumn{2}{|c|}{ M. tuberculous } & \multicolumn{2}{|c|}{ Non-tuberculous } & \multicolumn{2}{|c|}{ Culture negative } \\
\hline & No. & $(\%)$ & No. & $(\%)$ & No. & $(\%)$ & No & $(\%)$ \\
\hline $\begin{array}{l}\text { Distribution of the isolates } \\
\text { Gender }\end{array}$ & $(7 / 65)$ & 10.8 & $(27 / 65)$ & 41.5 & $(31 / 65)$ & 47.7 & $(392 / 457)$ & 85.8 \\
\hline Males & $(4 / 7)$ & 57.1 & $(12 / 27)$ & 44.4 & $(16 / 31)$ & 51.6 & $(184 / 379)$ & 48.5 \\
\hline Females & $(3 / 7)$ & 42.9 & $(15 / 27)$ & 55.6 & $(15 / 31)$ & 48.4 & $(195 / 379)$ & 51.5 \\
\hline \multicolumn{9}{|l|}{ Age } \\
\hline $1-19$ & $(4 / 7)$ & 57.1 & $(7 / 27)$ & 25.9 & $(11 / 31)$ & 35.5 & $(165 / 379)$ & 43.5 \\
\hline $20-39$ & $(3 / 7)$ & 42.9 & $(15 / 27)$ & 55.6 & $(14 / 31)$ & 45.2 & $(150 / 379)$ & 39.6 \\
\hline$>39$ & (0) & & $(5 / 27)$ & 18.5 & $(6 / 31)$ & 19.4 & $(64 / 379)$ & 16.9 \\
\hline HIV Positive & $(2 / 4)$ & $50^{\mathrm{a}}$ & $(1 / 10)$ & 10.0 & $(2 / 15)$ & 13.3 & $(13 / 124)$ & 10.5 \\
\hline History of $\mathrm{TB}$ in family & $(4 / 7)$ & 57.1 & $(10 / 26)$ & 38.5 & $(17 / 29)$ & 58.6 & $(168 / 360)$ & 46.7 \\
\hline Poor ventilation & $(1 / 7)$ & 14.3 & $(7 / 26)$ & 26.9 & $(6 / 29)$ & 20.7 & $(115 / 362)$ & 31.8 \\
\hline Livestock in the same house & $(1 / 4)$ & 25.0 & $(4 / 13)$ & 38.5 & $(8 / 24)$ & 33.3 & $(120 / 275)$ & 43.6 \\
\hline Drinking unboiled milk & $(4 / 7)^{b}$ & 57.1 & $(6 / 22)$ & 27.3 & $(9 / 28)$ & 32.1 & $(88 / 349)$ & 24.6 \\
\hline Uncooked animal products* & $(0 / 6)$ & 0 & $(7 / 26)$ & 26.9 & $(10 / 28)^{\mathrm{c}}$ & 35.7 & $(49 / 356)$ & 13.9 \\
\hline Adenitis sites & $(6 / 7)$ & 85.7 & $(22 / 26)$ & 84.6 & $(24 / 29)$ & 82.8 & $(320 / 359)$ & 89.1 \\
\hline \multicolumn{9}{|l|}{ Poor knowledge } \\
\hline TB spread animal to man & $(1 / 7)$ & 14.3 & $(8 / 26)$ & 30.8 & $(8 / 29)$ & 27.6 & $(103 / 353)$ & 29.2 \\
\hline TB spread by air drops & $(3 / 6)$ & 50.0 & $(16 / 26)$ & 61.5 & $(18 / 28)$ & 64.3 & $(240 / 339)$ & 70.8 \\
\hline
\end{tabular}

*Stratification analysis of the risk factors by district and tribes did not show statistically significant results. OR for HIV infection and status of boiling milk were adjusted for age and gender. OR for not cooking animal products adjusted for age. a OR (95\% C.I) = 13.63 ( 1.68 - 109.86), b OR $(95 \%$ C.I $)=15.28(3.26-71.69)$. ${ }^{\mathrm{c} O R}(95 \%$ C.1 $)=3.48(1.52-7.98)$. ${ }^{*}$ Blood, meat and offal.

Table 4

Model of multiple risk factors for mycobacterial isolates

\begin{tabular}{|c|c|c|c|c|c|c|}
\hline \multirow[t]{2}{*}{ Isolate } & \multicolumn{3}{|c|}{ Two risk factors* } & \multirow[b]{2}{*}{ OR } & More than & two risk factors* \\
\hline & OR & $95 \% \mathrm{Cl}$ & Model Chi-square & & $95 \% \mathrm{Cl}$ & Model Chi-square \\
\hline Culture positive & $3.5^{\mathrm{a}}$ & $1.4-9.0$ & $9.6(\mathrm{p}=0.008)$ & $4.2^{b}$ & $1.3-13.8$ & $14.0(\mathrm{p}=0.050)$ \\
\hline M. bovis & $23.6^{\mathrm{c}}$ & $1.9-299.3$ & $7.5(\mathrm{p}=0.024)$ & $16.2^{\mathrm{d}}$ & $1.3-201.3$ & $9.8(\mathrm{p}=0.021)$ \\
\hline Non-tuberculous & $4.5^{\mathrm{e}}$ & $1.3-15.4$ & $10.7(\mathrm{p}=0.005)$ & $5.2^{\mathrm{f}}$ & $1.3-20.6$ & $13.3(\mathrm{p}=0.010)$ \\
\hline Mycobacteria & & & & & & \\
\hline M. tuberculosis & $2.2 \mathrm{~g}$ & $0.6-8.4$ & $1.65(\mathrm{p}=0.44)$ & $2.2^{\mathrm{h}}$ & $(0.6-8.3)$ & $1.70(\mathrm{p}=0.79)$ \\
\hline
\end{tabular}


*HIV infection and uncooked animal products adjusted for sex and age. ahistory of TB in the family, and poor knowledge on transmission of TB from animals to humans

bhistory of TB in the family, drinking unboiled milk, uncooked animal products (blood, meat and offal), HIV infection and poor knowledge on transmission of TB from animals to humans

cHIV infection and unboiled milk,

$\mathrm{d}_{\mathrm{HIV}}$ infection, unboiled milk and poor ventilation history of TB in the family, and poor knowledge on transmission of TB from animals to humans

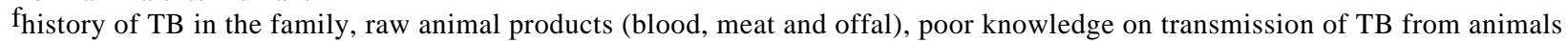
to humans, and HIV infection

gPoor ventilation, history of TB in the family, poor ventilation, HIV infection, history of TB in the family, poor knowledge on TB spread by infected air drops

This set of three factors, together, had an OR of 16.2 (95\%CI, 1.3 - 201.3). The patients of non-tuberculous mycobacteria adenitis were linked to a set of five risk factors $(O R=5.2,95 \% \mathrm{CI}, 1.3-20.6)$. The factors were HIV infection, drinking raw milk, eating raw animal products (blood, meat and offal) and poor knowledge on transmission of TB from animals to humans in a patient with history of TB in the family. The assessments did not show significant association to any of the risk factors for adenitis caused by $M$. tuberculosis.

\section{DISCUSSION}

Several studies have been done to investigate human tuberculosis as a main cause of human morbidity and mortality in Tanzania. However, a few studies have focused on mycobacterial adenitis $(9,13)$ caused by $M$. bovis $(9)$.

The present study shows that $M$. bovis causes at least one out of ten cases of mycobacterial adenitis. This indicates that $M$. bovis indeed play a role in mycobacterial adenitis in Tanzania. It is so, especially, in parts of the country where traditional farming and livestock keeping is common. Traditional farmers and livestock keepers are the main inhabitants of the region where this study was conducted.

Although $M$. bovis caused a relatively small proportion of mycobacterial adenitis as compared to $M$. tuberculosis and non-tuberculous mycobacteria, it is worrisome because of report of the disease in livestock and wild animals (14, 15, DFID Report, R7229/R7357, 2002), and its link with HIV infection. Tuberculin surveys in cattle indicate a prevalence of the infection of about $13 \%$, and a herds' prevalence ranging from $15 \%$ to $51 \%$ in Tanzania (14, 15, DFID Report, R7229/ R7357, 2002). Besides, trading of livestock products between rural and urban areas is increasing. The trading raises a special concern because $M$. bovis has been isolated from specimens obtained from milk $(6.5 \%$ of 31 specimens), slaughtered cattle in slaughterhouses and wildlife (5 out of 10 isolates were M. bovis). Wildebeest and buffalos were among the wildlife infected with $M$. bovis. Survey of meat inspections in slaughterhouses showed viable lesions in $25 \%$ of cattle slaughtered from Hanang, 23\% from Mbulu, 12\% from Babati and 5.6\% from Karatu. Of 241 positive cultures from slaughterhouses specimens (1620), $16.2 \%$ were $M$. bovis (DFID Report, R7229/R7357, 2002).
While HIV prevalence in tuberculosis patients varies from $21 \%$ to $77 \%$ within various regions of the Tanzania, a prevalence of $25 \%$ have been reported from the study region(8). Mycobacterial adenitis is not directly infectious, but some cases of compromised immunity might develop into disseminated or reactivated disease and become infectious. Findings at autopsy in two studies in Zaire and Cote d'Ivoire showed tuberculosis in $32 \%$ of HIV-positive cadavers. In $87 \%$ of cadavers, TB was disseminated to more than one organ, including lymph nodes and nearly always involving the lung(16). However, the control challenges of $M$. bovis adenitis, upcoming as consequences of the HIV epidemic, are not fully understood. One pulmonary case of a dual HIV and M. bovis infection that was resistant to several antituberculous drugs in a Paris hospital was reported to infect five other patients in less than 10 months (17). O'Reilly and Darborn(3), indeed gives a clear review of the potential threat posed by the HIV pandemic to an increase of human tuberculosis due to $M$. bovis infection in the world. It is therefore disturbing knowing that there is no clear policy for control strategy of bovine tuberculosis.

The NTLP in Tanzania has reported treatment success rate of about $80 \%$ and low level of drug resistance. This is a good achievement nation-wide (NTLP annual reports, 1984-2000). However, the outcome of treatment for extra-pulmonary cases is not based on microbacteriological findings. In addition, culture from biopsy tissues and $M$. bovis identification are not done routinely, and therefore the contribution of $M$. bovis infection to the resistance pattern in the country is not known. A clear control policy for this infection will benefit communities involved in livestock keeping in Tanzania as well as in other developing countries.

The control policy should have a public education package that will focus on the existing risk factors linked to the infection. The present study emphasises the role of consuming raw animal products (eating raw blood, meat and offal) and HIV infection in causation of $M$. bovis adenitis in this area. This is shown by the risk posed by these factors. Houses with inadequate ventilation might enhance aerosol transmission of $M$. tuberculosis as well as M. bovis from cattle to cattle or from cattle to humans. The risk of inadequate ventilation was linked to $M$. bovis adenitis in modelling risk assessment. Although this study was not able to 
link the risk posed by inadequate ventilation and HIV infection to adenitis caused by $M$. tuberculosis, the linkage should not be ruled out because of small sample size of the isolates involved in comparison analysis.

The current study raises another concern on the finding that non-tuberculous mycobacteria were as common as $M$. tuberculosis in causation of mycobacteria adenitis. The disease with culture-positive mycobacteria was found in about $34.0 \%(22 / 65)$ of childhood and adolescence cases, ageing between 1 and 19 years. Recently, non-tuberculous mycobacterial adenitis has been linked to cervicofacial manifestations in childhood, adolescence and HIV infection $(5,6)$.

The current findings suggest a possibility of being infected by ingesting raw animal products (including milk). HIV infection, history of TB in family and poor knowledge on how TB spreads increase the possibility of the infection. Of 31 samples of infected milk from Southern Highlands of Tanzania, $87 \%$ were nontuberculous mycobacteria. The non-tuberculous mycobacteria included $M$. terrae, $M$. fortuitum, M. flavescens, M. gordonae and M. smegmatis(18). The same mycobacteria, and $M$. avium intracellular complex were also isolated in human specimens in the current study area (DFID Report, R7229/R7357, 2002). Furthermore, the same non-tuberculous mycobacteria were isolated in most of specimens collected from slaughterhouses and wildlife in the same study area (DFID Report, R7229/R7357, 2002). This area has several national parks and game reserves, with a number of wild ruminants. The ruminants are equally susceptible to zoonotic $\mathrm{TB}$, as it has also been demonstrated in South Africa(19).

Considering these facts, the infection due to nontuberculous mycobacteria raises a concern to human health, especially, in childhood and people with poor immunity due to HIV infection. The incidence of HIV infection is increasing in most of developing countries such as Tanzania. The current study was able to link HIV infection to non-tuberculous mycobacterial infection in assessment of multiple risk factors.

The risk of history of tuberculosis contact in the family to culture positivity, indicate that risk factors are shared within the family. This has also been shown in Malawi by a higher frequency of TB in households of index TB patients(20). Public education on how mycobacterial adenitis is acquired and how it can be avoided may prevent future spread of the disease. Poor knowledge, especially on transmission of TB from animals to humans, is a risk factor for acquiring culture positive adenitis due to non-tuberculous mycobacteria. The education could have a higher potential benefit to the patients from Barabaig and Iraw tribes as well as Mbulu and Hanang districts. The groups were at increased risk of acquiring mycobacteria adenitis through eating or drinking raw animal products as suggested by culture positive results. Therefore, any control policy should give priority to these groups. Strategies involving collaboration between NTLP and AIDS control programmes need further investigation. More information on occurrence of M. bovis and non-tuberculous mycobacterial disease in humans, especially in pulmonary site, and resistance pattern to antituberculous drugs is required, if any advice on management policy is to be given. An in-built surveillance system in the existing laboratory network should be able to establish the information required.

It is, however, important to realise the possibility for underestimating the risk factors assessed in this study. Some study patients might have been aware of medical advice against habits that are linked to spread of the disease. Such patients might be inclined to give a biased information to fit the social and medical desirability. Culture is a definitive diagnosis of mycobacterial disease. However, most of patients meeting clinical criteria for mycobacterial adenitis were culture negative. In addition to specificity problem of the clinical criteria, the viability of mycobacterial cultures is also known to vary with conditions, including number of mycobacteria in specimens. It is known that the numbers of mycobacteria are low in typical granulomatous response(16). Therefore, it is likely that some patients might have been improperly classified as culture negative cases. The potential misclassification might have caused underestimation of the risk factors.

Comparison of tuberculin tests in cattle in both groups could give more information regarding $M$. bovis infection. A control group with baseline characteristic of the general population would reflect estimations that are more representative of the general population.

\section{CONCLUSIONS}

Mycobacterium bovis caused one out of ten cases of culture positive mycobacterial adenitis. Nontuberculous mycobacteria were more common than $M$. tuberculosis (50\% and $40 \%$ of the cases, respectively). HIV infection was a risk factor for $M$. bovis and nontuberculous mycobacterial adenitis. Other identified risk factors for $M$. bovis were associated with nontuberculous mycobacteria as well. The factors were history of TB in the family, drinking raw milk, eating raw animal products, poor ventilation, and having poor knowledge about transmission of tuberculosis. The patients from Iraqw and Barabaig tribes as well as from Hanang and Mbulu districts had higher risk of mycobacterial adenitis than the other groups.

\section{ACKNOWLEDGEMENTS}

To the Norwegian Research Council, Norway and Department for International Development, UK for funding this study. We are grateful to the Central Unit of NTLP and Tuberculosis Co-ordinators in the study area for their contribution in data collection and management of the patients. The authors also wish to thank Said Mfaume and all other laboratory technicians for their tireless effort in processing of the specimens. 


\section{REFERENCES}

1. Cosivi, O., Grange, J.M., Daborn, C. J. et al. Zoonotic tuberculosis due to mycobacterium bovis in developing countries. Emer. Infect. Dis. 1998; 4:59-73.

2. World Health Organisation. Zoonotic tuberculosis (Mycobacterium bovis): Memorandum from a WHO meeting (with participation of FAO). Bull. World Hlth. Org. 1994; 72:851-857.

3. O'Reilly, L.M. and Daborn, C. J. The epidemiology of Mycobacterium bovis infection in animals and man: a review. Int. J. Tuberc. Lung. Dis. 1995; 76: (Suppl. 1) 1-46.

4. Kleeberg, H.H. Human tuberculosis of bovine origin in relation to public health. Rev. Sci. Tech. Off. Int. Epiz. 1984; 3:11-32.

5. Evans, M.J., Smith, N.M., Thornton, C.M., Young-son, G.G., and Gray, E.S. Atypical mycobacterial lymphadenitis in childhood-a clinicopathological study of 17 cases. J. Clin. Pathol. 1998; 51:925-927.

6. Zumla, A., and Grange, J. Infection and disease caused by environmental mycobacteria. Curr. Opinion in Pulm. Med. 2002; 8:166-172

7. Venkatesh, V., Everson, N.W., and Johnstone, J.M. Atypical mycobacterial Iymphadenopathy in children. Is it underdiagnosed? J. R. Coll. Surg. Edinb. 1994; 39:301-303.

8. Range, N., Ipuge, Y.A., O'brien, R. J et al. Trend in HIV prevalence among tuberculosis patients in Tanzania, 19911998. Int. J. Tuberc. Lung. Dis. 2001; 5:405-412.

9. Kazwala, R. R.; Daborn, C. J., Sharp, J. M., et al. Isolation of Mycobacterium bovis from human cases of cervical adenitis in Tanzania: a cause for concern? Int. J. Tuberc. Lung. Dis. 2001; 5:87-91.

10. Grange, J.M., and Collins, C.H. Bovine tubercle bacilli and disease in animal and man. Epidem. Infect. 1987; 99:221-234.

11. Collins, C.H. and Grange, J.M. A review: the bovine tubercle bacillus. J. Appl. Bacter. 1983; 55:13-29.
12. Norusis, M. J. SPSS for Windows Advanced Statistics Version 7.5. SPSS In: Logistic regression analysis. Inc, Chicago, IL, 1996: pp 1-30.

13. Perenboom, P.M., Richter, C., Swai, A.B.M. et al. Clinical features of HIV seropositive and HIV seronegative patients with tuberculous Iymphadenitis in Dar-es Salaam. Tuberc. Lung Dis. 1995; 76:401-406.

14. Jiwa, S.F., Kazwala, R.R., Aboud, A.A., and Kalaye, W.J. Bovine tuberculosis in the Lake Victoria zone of Tanzania and its possible consequences for human health in the HIV/AIDS era. Vet. Res. Comm. 1997; 21:533-539.

15. Kazwala, R.R., Kambarage, D. M., Daborn, C.J., Nyange, J., Jiwa, S.F., and Sharp, J.M. Risk factors associated with the occurrence of bovine tuberculosis in cattle in the Southern Highlands of Tanzania. Vet. Res. Comm. 2001; 25;609-614.

16. Barry, R.B. Tuberculosis. In: Lucas S. and Nelson A. M. ed. Pathogenesis of tuberculosis in human immunodeficiency virus-infected people. American society for microbiology, Washinton, DC,. 1994; 25-46.

17. Bouvet, E., Casalino, E., Mendoza-Sassi G., et al. A nasocomial outbreak of muliti- drug- resistant Mycobacterium bovis among HIV infected patients. A case control study. AIDS. 1993; 7:1453-1460.

18. Kazwala, R.R, Daborn, C.J. Kusiluka, L.J, et al. Isolation of Mycobacterium species from raw milk of pastoral cattle of the Southern Highlands of Tanzania. Trop. Animal Hlth Prod. 1998; 30:233-239.

19. De Vos, V. Bengis, R. G. Kriek, N.P., et al. The epidemiology of tuberculosis in free-ranging African buffalo (Syncerus caffer) in the Kruger National Park, South Africa. Onderstepoort J. Vet. Res. 2001; 68:119130.

20. Cleassens, N.J.M. Gausi, F.F, Meijne, S. et al. High frequency of tuberculosis in households of index TB patients. Int. J. Tuberc Lung Dis. 2002; 6:266-269. 